\title{
Development of a diet quality index assessing adherence to the Swedish nutrition recommendations and dietary guidelines in the Malmö Diet and Cancer cohort
}

\author{
Isabel Drake* + , Bo Gullberg, Ulrika Ericson, Emily Sonestedt, Jan Nilsson, Peter \\ Wallström, Bo Hedblad and Elisabet Wirfält \\ Department of Clinical Sciences in Malmö, Lund University, Skåne University Hospital, Malmö, Sweden
}

Submitted 9 June 2010: Accepted 13 December 2010: First published online 7 February 2011

\begin{abstract}
Objective: To develop a diet quality index (DQI) that assesses adherence to the Swedish nutrition recommendations (SNR) and the Swedish dietary guidelines (SDG).

Design: A cross-sectional study within the Malmö Diet and Cancer (MDC) cohort. A diet history method collected dietary data, a structured questionnaire lifestyle and socio-economic information, and anthropometric data were collected by direct measurements. The index (DQI-SNR) included six components: SFA, PUFA, fish and shellfish, dietary fibre, fruit and vegetables, and sucrose.

Setting: Malmö, Sweden.

Subjects: Men ( $n$ 4525) and women ( $n$ 8491) of the MDC cohort enrolled from September 1994 to October 1996.

Results: For participants with high DQI-SNR scores, nutrient and food intakes were close to recommendations. However, most of the study population exceeded the recommended intake for SFA (98\%) and few reached recommended intakes for dietary fibre (24\%), fruit and vegetables (32\%), vitamin D (18\%) and folate (2\%). A high DQI-SNR score was positively associated with age, physical activity, not smoking, past food habit change, education and socio-economic status. Individuals with high scores were more likely to have a diabetes diagnosis or experienced a cardiovascular event.

Conclusions: Results suggest that the DQI-SNR is a useful tool for assessing adherence to the SNR 2005 and the SDG in the MDC cohort. No index has previously been developed with the aim of evaluating adherence to the current dietary recommendations in Sweden. Further validation of the DQI-SNR, and evaluation of its utility, is needed.
\end{abstract}

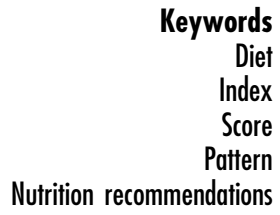

The complex nature of diet, with specific combinations of foods and nutrients, impedes nutrition epidemiology when investigating diet-disease associations; therefore a dietary pattern approach may be more appropriate when investigating disease outcomes ${ }^{(1-7)}$. Diet quality indices consist of a combination of foods and/or nutrient components that together represent dietary guidelines ${ }^{(8-10)}$. The use of index-based dietary patterns is one way to address the complexity of diet, while at the same time evaluating the effectiveness of current dietary guidelines to prevent chronic diseases ${ }^{(11)}$. Recent reviews have reported more than twenty indices currently in existence ${ }^{(10-14)}$. Studies using dietary indices have found associations with

$\dagger$ Correspondence address: Lund University, Department of Clinical Sciences in Malmö, Nutrition Epidemiology, CRC entrance 72 building 60 level 13, SE-205 02 Malmö, Sweden. nutrient adequacy ${ }^{(15-18)}$, biomarkers of disease ${ }^{(19-21)}$, premature mortality ${ }^{(22-26)}, \mathrm{CVD}^{(27,28)}$ and certain forms of cancer $^{(29,30)}$. However, few previously constructed indices are suitable for direct application in a Swedish population since most include foods not typically consumed in Swedish diets or are based on dietary guidelines different from those in Sweden.

The official Swedish dietary guidelines consist of the Swedish nutrition recommendations (SNR) 2005 and the Swedish dietary guidelines (SDG) ${ }^{(31,32)}$. The SNR 2005 are based on the Nordic Nutrition Recommendations 2004 (NNR 2004) and include recommendations for the macronutrient composition of the diet, the daily micronutrient intake, and reference values for energy intake ${ }^{(33)}$. The NNR 2004 are based on available scientific evidence and provide a basis for evaluating the intake of nutrients in groups of healthy individuals ${ }^{(33)}$. The SDG are food-based 
dietary guidelines published by the Swedish National Food Administration which communicate the concept of a diet that fulfils the SNR $2005^{(32)}$. In order to illustrate the practical application of the SNR 2005, the Swedish Nutrition Recommendations Objectified (SNO) project developed a food intake pattern for adults based on seventy-one foods considered to be representative of Swedish eating habits ${ }^{(34)}$. A comparison between SNO and the most recent national dietary survey, Riksmaten 1997/98, formed the basis for the main SDG, which aim at increasing the population's intakes of fruit and vegetables, wholegrain products, fish and vegetable oils, and limiting intakes of animal fat, sugar and salt ${ }^{(32,35)}$.

Despite increased public awareness of the importance of diet in decreasing the risk of chronic disease, large gaps remain between current recommendations and actual dietary practices in Sweden ${ }^{(35,36)}$. The nutritional problems in Sweden relate mainly to a macronutrient distribution with too much fat (especially saturated fat), refined sugar and alcohol, and too little complex carbohydrates and dietary fibre. Micronutrient intakes are generally sufficient apart from deficient intakes of vitamin D, folate and Fe in certain subgroups of the population, and an overconsumption of salt ${ }^{(33,35,36)}$.

The aim of the present study was to develop and evaluate a diet quality index (DQI) that may be used as a tool to distinguish high- and low-quality diets by assessing adherence to the SNR 2005 and SDG.

\section{Materials and methods}

\section{Study population}

The Malmö Diet and Cancer (MDC) study is a populationbased prospective cohort ( $n$ 28 098). Baseline examinations were conducted between 1991 and 1996, and eligible participants were men born between 1923 and 1945 and women born between 1923 and 1950, living in the city of Malmö and with Swedish reading and writing skills. The MDC study was approved by the Ethical Committee at the Medical Faculty, Lund University (LU 51-90). Details of the recruitment procedures and the cohort are given elsewhere $^{(37,38)}$. The data collection included dietary habits, socio-economics, medical history and lifestyle habits using questionnaires and interview. Halfway through the baseline data collection, a change of coding routines was implemented in order to reduce interview time. For descriptive studies it has therefore been recommended to use the subset of the population that completed the baseline examinations after this revision ${ }^{(39)}$. Thus the present study is based on participants, men ( $n$ 4525) and women ( $n$ 8466), enrolled in the MDC study after 1 September 1994 (total $n$ 12991).

\section{Dietary assessment}

Information on dietary habits was obtained through a modified diet history method combining a $7 \mathrm{~d}$ menu book (collecting descriptions of prepared meals, nutrient supplements and cold beverages) and a 168-item quantitative diet questionnaire using both exact frequencies and a picture booklet to assess portion sizes of regularly eaten foods other than prepared meals during the past year. During a $1 \mathrm{~h}$ interview, the questionnaire and the menu book were checked so that reported food consumption did not overlap and detailed information was collected on cooking practices and recipes. Energy and nutrient intakes were computed from the reported food intake using the MDC Food and Nutrient Database, originating mainly from PC Kost2-93 of the National Food Administration in Uppsala, Sweden ${ }^{(40)}$. Data on the validity ${ }^{(41,42)}$ and reproducibility ${ }^{(43)}$ of the method have been published. The relative validity of the MDC method is high compared with other dietary assessment methods in similar populations ${ }^{(44,45)}$.

\section{Socio-economic and lifestyle variables}

Information on socio-economic and lifestyle factors was collected from the extensive lifestyle and socio-economic questionnaire. A high educational level was defined in the present study as upper secondary school or higher (more than 11 years). The smoking habits of the participants were defined as current smokers (including irregular smokers), former smokers or never smokers. Leisure-time physical activity was assessed by a list of activities in the questionnaire, modified from the Minnesota Leisure Time Physical Activity Questionnaire ${ }^{(46-48)}$. Participants were asked to report how many minutes per week on average, and for each of the four seasons, they spend on a specific activity. A physical activity score was obtained by multiplying the number of minutes for each activity with an activity-specific factor. A high leisure-time physical activity was defined as individuals in the highest genderspecific tertile of activity score. Physical activity at work self-rated by participants as very light or light was defined as sedentary work. Alcohol habits were classified as zero, low, moderate or high consumption. Participants reporting no alcohol intake in the $7 \mathrm{~d}$ menu book and reporting no alcohol intake during the preceding year in the questionnaire were classified as zero alcohol consumers. For all other participants low, medium and high alcohol consumption level was set at alcohol intakes of $<15$, $15-30$ and $>30 \mathrm{~g} / \mathrm{d}$ for women, and $<20,20-40$ and $>40 \mathrm{~g} / \mathrm{d}$ for men. Mattisson et al. ${ }^{(49)}$ have previously defined low, adequate and high energy reporters in the MDC cohort using the approach described by Goldberg et $a l^{(50)}$ and later refined by $\mathrm{Black}^{(51)}$. Energy misreporting was defined as having a ratio of energy intake to BMR outside the $95 \%$ CI limits of the calculated physical activity level. Past food habit change was based on the questionnaire item 'Have you substantially changed your dietary habits because of illness or another reason?' Previous cardiovascular event (including coronary event or stroke) was determined though local registers and diabetes diagnosis was self-reported by the participants. 
Table 1 Summary of the Swedish nutrition recommendations (SNR 2005) and the Swedish dietary guidelines (SDG)

\begin{tabular}{|c|c|}
\hline Guidance/component & Description \\
\hline \multicolumn{2}{|l|}{ SNR 2005} \\
\hline \multicolumn{2}{|l|}{ Fat } \\
\hline Total fat & $25-35 \% \mathrm{E}$ \\
\hline SFA and trans fatty acids & $<10 \% \mathrm{E}$ (intake of trans fatty acids should be kept as low as possible) \\
\hline MUFA & $10-15 \% \mathrm{E}$ \\
\hline PUFA & $5-10 \% \mathrm{E}$ (approximately $1 \% \mathrm{E}$ from $n-3$ fatty acids) \\
\hline Essential fatty acids ( $n-6$ and $n-3)$ & $>3 \% \mathrm{E}$ (including at least $0.5 \% \mathrm{E}$ from $n-3$ fatty acids \\
\hline \multicolumn{2}{|l|}{ Carbohydrates } \\
\hline Total carbohydrates & $50-60 \% \mathrm{E}$ \\
\hline Dietary fibre & $25-35 \mathrm{~g} / \mathrm{d}$ (equivalent to approximately $3 \mathrm{~g} / \mathrm{MJ}$ ) \\
\hline Added sugars & $<10 \% \mathrm{E}$ \\
\hline \multicolumn{2}{|l|}{ Protein } \\
\hline Total protein & $10-20 \% E$ \\
\hline Vitamins and minerals & Daily recommended intakes are available in SNR $2005^{(31)}$ \\
\hline Salt & $6 \mathrm{~g} / \mathrm{d}$ for women; $7 \mathrm{~g} / \mathrm{d}$ for men \\
\hline Alcohol & $<5 \% \mathrm{E}(10 \mathrm{~g} / \mathrm{d}$ for women; $20 \mathrm{~g} / \mathrm{d}$ for men $)$ \\
\hline \multicolumn{2}{|r|}{ 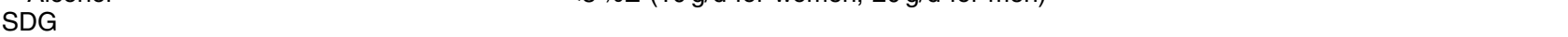 } \\
\hline Fruits and vegetables & $500 \mathrm{~g} / \mathrm{d}$ (at least $400 \mathrm{~g} / \mathrm{d}$ excluding juices) \\
\hline Fish and shellfish & $2-3$ times weekly (about $300 \mathrm{~g}$ raw fish, partly fatty) \\
\hline Dietary fats and oils & Liquid margarine or oil in food preparation \\
\hline Dry legumes & At least $50 \mathrm{ml}$ cooked legumes daily ( $10 \mathrm{~g}$ dry) \\
\hline Cereals, rice and potatoes & $\begin{array}{l}\text { 1-2 portions daily from potato, rice or pasta, bread or cereals to each meal, corresponds } \\
\text { to } 200-250 \mathrm{~g} \text { cereals/grains daily; preferably wholegrain }\end{array}$ \\
\hline Milk & $\frac{1}{2}$ litre low-fat milk/yoghurt daily, may partly be exchanged with low-fat cheese \\
\hline Meat and meat products & $140 \mathrm{~g} \mathrm{low}$-fat meat or meat products daily \\
\hline Discretionary foods (primarily sweet foods) & $\begin{array}{l}\text { Intake of snacks, ice cream, cakes, pastries, biscuits, sweets, chocolate, soft drinks and } \\
\text { jam should be minimized and constitute no more than } 10 \% \mathrm{E}\end{array}$ \\
\hline
\end{tabular}

$\% \mathrm{E}$, percentage of energy.

\section{Antbropometric variables}

Nurses measured height $(\mathrm{m})$, weight $(\mathrm{kg})$, waist and hip circumferences $(\mathrm{cm})$. BMI was calculated as weight divided by the square of height $\left(\mathrm{kg} / \mathrm{m}^{2}\right)$. Blood pressure $(\mathrm{mmHg})$ was measured after $10 \mathrm{~min}$ of rest.

\section{Development of the diet quality index (DQI-SNR)}

Index component selection

The SNR and SDG are overlapping and complementary, and therefore both were considered for identification of suitable index components (Table 1). Three main aspects were considered during the selection process. First, information on dietary intake of candidate components had to be available within the MDC cohort. Initial assessment therefore excluded trans fatty acids and salt intake, since information on these dietary factors was either lacking or had poor validity. Second, dietary components considered most important in assuring overall diet quality (i.e. food groups and macronutrients) in relation to chronic disease were primarily considered. Third, inter-correlation between components was investigated to assess the mutual independence between components ${ }^{(10,14)}$.

Considering these three aspects, the following components were selected: SFA, PUFA, fish and shellfish, dietary fibre, fruit and vegetables, and sucrose. SFA and PUFA were selected to reflect the fat quality of the diet as well as reflecting the recommendation to use liquid margarine or vegetable oils in food preparation. There is a strong correlation between the subtypes of fat in the MDC cohort. The strong correlation $(r=0.65)$ between MUFA and SFA in the MDC cohort suggests that the dietary sources of these two fats are mainly the same (i.e. from meat and dairy products). This has recently been highlighted by a pooled analysis of dietary fat and $\mathrm{CHD}^{(52)}$. Therefore, MUFA was not included as a component. In addition, SFA and PUFA jointly reflect total fat intake and low SFA intake is also likely to reflect a preference for low-fat dairy and low-fat meat products. The different subtypes of PUFA were similarly highly correlated $(r=0.97)$ and total PUFA was therefore selected as an index component, as it is likely to reflect intakes of both $\alpha$-linolenic acid and linoleic acid. Dietary fibre reflects intake of high-fibre cereals (i.e. whole grains; $r=0 \cdot 39$ ) and fibre from fruit and vegetables $(r=0.63)$. The foodbased dietary guideline for increasing dietary fibre intake by choosing fibre-rich foods was recently changed to selecting primarily wholegrain cereals. However, there is no specified amount of recommended whole grains intake; therefore dietary fibre was included as a component to reflect this aspect of diet. Sucrose was included to reflect intake of refined sugars and consumption of sugarrich foods. Table 2 shows a description of the DQI-SNR.

\section{Cut-offs and scoring}

A cut-off for each index component was assigned based on the recommended level of intake in the SNR 2005 or the SDG (see Table 1). A score of 1 was assigned to individuals complying with the recommendation and a score of 0 was assigned to those not complying with the 
Table 2 DQI-SNR: componentst, cut-offsł and differences in component adherence (\%) between men ( $n$ 4525) and women ( $n$ 8491) of the Malmö Diet and Cancer cohort

\begin{tabular}{|c|c|c|c|c|}
\hline \multicolumn{2}{|c|}{ Description of DQI-SNR } & \multicolumn{3}{|c|}{ Adherence } \\
\hline Index component & Cut-off & Men & Women & $P \S$ \\
\hline SFA & $\leq 14$ & $23 \cdot 3$ & $24 \cdot 4$ & 0.189 \\
\hline PUFA & $5-10$ & $73 \cdot 0$ & $66 \cdot 0$ & $<0.0001$ \\
\hline Fish and shellfish (g/week) & $\geq 300$ & $48 \cdot 4$ & $40 \cdot 8$ & $<0.0001$ \\
\hline Dietary fibre $(\mathrm{g} / \mathrm{MJ})$ & $2 \cdot 4-3 \cdot 6$ & $17 \cdot 1$ & $31 \cdot 0$ & $<0.0001$ \\
\hline Fruit and vegetables $(\mathrm{g} / \mathrm{d})$ & $\geq 400$ & $26 \cdot 1$ & $38 \cdot 0$ & $<0.0001$ \\
\hline Sucrose & $\leq 10$ & $71 \cdot 8$ & $67 \cdot 7$ & $<0.0001$ \\
\hline
\end{tabular}

DQI-SNR, diet quality index based on the Swedish nutrition recommendations 2005.

tExpressed as non-alcohol energy percentage (\%E) unless otherwise noted.

¥Adherence provides 1 point to total score, non-adherence 0 points.

$\$ P$ from $\chi^{2}$ test. Values for non-adherence are not shown.

recommendation. A total score was created by summing the score of each component, thus total score ranged from 0 to 6 . Using predefined cut-offs can cause problems when the population has intake levels that are not close to the recommendation. This was the case for SFA, where only $2 \%$ of the MDC population complied with the recommendation ( $\leq 10 \%$ of energy (\%E)). Therefore, the cut-off for SFA was defined by adding one standard deviation of the mean population intake to the recommended level, resulting in a new cut-off $(\leq 14 \% \mathrm{E})$. The recommendation for dietary fibre intake is $25-35 \mathrm{~g} / \mathrm{d}$ (approximately $3 \mathrm{~g} / \mathrm{MJ}$ ). The energy relative recommendation has no defined upper or lower limit, and therefore a similar approach was applied for the dietary fibre component (i.e. \pm 1 sD of the population mean). The fruit and vegetable component excludes fruit juices and the cut-off was therefore set at $\geq 400 \mathrm{~g} / \mathrm{d}$, instead of the recommended $\geq 500 \mathrm{~g} / \mathrm{d}$ (including a maximum of $100 \mathrm{~g}$ fruit juices).

\section{Evaluation of the diet quality index (DQI-SNR)}

It is important that the index assesses diet quality independent of diet quantity. Because most nutrient intakes are positively correlated with energy intake, a diet quality index could overrate high-energy diets if intakes are measured in terms of absolute amounts. In nutrition epidemiology, energy-adjusted variables ensure the isoenergetic principle and in addition reduce the impact of measurement errors associated with self-reported data ${ }^{(53)}$. Recommendations for most of the index components are expressed in energy relative terms $(\% \mathrm{E}$ or $\mathrm{g} / \mathrm{MJ})$. However, fish intake and fruit and vegetable intake are expressed as gram per week or $\mathrm{d}$. Energy independence was therefore investigated by examining correlations between components, total score and total energy intake. In addition, since alcohol intake tends to dilute diet composition and has established health effects, we used non-alcohol energy intake for calculation of energy percentage $(\% \mathrm{E})$.

The face validity of the index, i.e. quantitative evaluation of how well the index measures what it is supposed to measure ${ }^{(54)}$, was assessed in several ways. First, the correlation between the total score and the index components was investigated to assess the relative weight of components. Second, we examined how total score was associated with nutrient intakes and selected food groups. The nutrient variables investigated in the study included total energy intake and relative intakes of fat (total fat, SFA, MUFA, PUFA, $n-6$ and $n-3$ fatty acids), carbohydrates (including $\% \mathrm{E}$ from total carbohydrates and dietary fibre intake), protein and alcohol. The intakes of selected vitamins and minerals (including retinol equivalents, vitamin D, vitamin $\mathrm{E}$, thiamin, riboflavin, niacin equivalents, vitamin $\mathrm{B}_{6}$, folic acid, vitamin $\mathrm{B}_{12}$, ascorbic acid, Ca, P, K, Mg, Se, Zn and $\mathrm{Fe}$ ) were assessed by investigating the proportion of the study sample reaching the recommended intake levels in the NNR $2004(\mathrm{~g} / \mathrm{MJ})^{(33)}$. In addition, intakes of fruit and berries, vegetables, fish and shellfish, butter and margarines, cereals, high-fibre cereals, potatoes, rice and pasta, high- and low-fat dairy, high- and low-fat meat, soft drinks, cakes and biscuits, and sweets and chocolate were examined. Third, we examined the concurrent-criterion validity; whether the index can distinguish between groups of people with different lifestyle and socio-economic characteristics that have previously been observed to report diets of diverging quality ${ }^{(54)}$.

\section{Statistical analyses}

The proportion of participants meeting each component cut-off was calculated and the $\chi^{2}$ test was used to compare differences between men and women. Pearson partial correlations were estimated to evaluate inter-correlation among the index components as well as the correlation between index components, energy intake and total score; analysis was adjusted for age, sex and season (autumn, winter, spring, summer). Mean intakes of the DQI-SNR components by total index score were assessed while adjusting for age, season and total energy intake. Because there were few individuals in the low and high DQI-SNR score subgroups, subsequent analyses investigated categories of DQI-SNR score, i.e. low score ( 0 or 1 point), medium score ( 2 or 3 points) and high score ( $4-6$ points). Mean nutrient and food intakes were investigated across categories of DQI-SNR score and linear trends across continuous DQI-SNR score were calculated adjusting for age, season and total energy intake. Associations between DQI-SNR score and participant characteristics were investigated using the linear regression coefficient for the DQI-SNR score for continuous variables and the logistic regression coefficient for the DQI-SNR score for dichotomous variables, while adjusting for age and total energy intake. Additional adjustments were made for leisure-time physical activity for anthropometric characteristics and for leisure-time physical activity and BMI for disease history characteristics. The SPSS statistical software package version 17 (SPSS Inc., Chicago, IL, USA) was used for all statistical analyses; $P<0 \cdot 05$ was considered significant. 


\section{Results}

Table 2 describes the construction of the DQI-SNR and shows the percentage of men and women adhering to the selected cut-offs. Table 3 shows the correlation coefficients for components, total score and total energy intake. Most components showed low to intermediate intercorrelation. Dietary fibre was negatively correlated with SFA $(r=-0.52)$. There was also a positive correlation between fruit and vegetables and dietary fibre $(r=0.63)$. Total score showed energy independence $(r=-0 \cdot 04)$; however, there was some correlation between the individual components and total energy intake. Correlations between individual components and total score ranged from $|r=0 \cdot 21|$ to $|r=0 \cdot 53|$.
Population median intake and interquartile range of the DQI-SNR components are shown in Table 4 , along with mean intakes of the components across total index score. All trends across score were significant $(P<0 \cdot 0001)$. Mean relative intakes of macronutrients across categories of DQI-SNR score (low, medium and high) are shown in Table 5. All trends were significant with $P<0 \cdot 0001$; however, the trends were not as clear as for all macronutrients (i.e. total fat and total carbohydrate). Figures 1(a) and 1(b) respectively show the mean intakes of different food groups in the categories of DQI-SNR score among men and women. Participants with a high DQI-SNR score had significantly higher intakes of fruit and berries, vegetables, fish and shellfish, margarines, cereals, highfibre cereals, low-fat dairy products and meat (all $P$ for

Table 3 Partial correlationst between DQI-SNR componentsł, total score and total energy intake among men and women $(n 12991)$ of the Malmö Diet and Cancer cohort

\begin{tabular}{|c|c|c|c|c|c|c|c|c|}
\hline & Energy & SFA & PUFA & Fish and shellfish & Dietary fibre & Fruit and vegetables & Sucrose & Total score \\
\hline Energy & 1.00 & & & & & & & \\
\hline SFA & $0.28^{*}$ & 1.00 & & & & & & \\
\hline PUFA & $0.03^{*}$ & $-0.06^{\star}$ & $1 \cdot 00$ & & & & & \\
\hline Fish and shellfish & $0 \cdot 16^{*}$ & $-0.06^{\star}$ & $0 \cdot 14^{*}$ & 1.00 & & & & \\
\hline Dietary fibre & $-0.22^{*}$ & $-0.52^{*}$ & $-0 \cdot 12^{\star}$ & $0.03^{*}$ & $1 \cdot 00$ & & & \\
\hline Fruit and vegetables & $0 \cdot 18^{*}$ & $-0.28^{\star}$ & $-0.08^{*}$ & $0 \cdot 14^{*}$ & $0.63^{*}$ & $1 \cdot 00$ & & \\
\hline Sucrose & $0 \cdot 15^{\star}$ & $-0 \cdot 18^{\star}$ & $-0 \cdot 20^{\star}$ & $-0.08^{*}$ & $-0 \cdot 13^{\star}$ & $0.04^{\star}$ & $1 \cdot 00$ & \\
\hline Total score & $-0.04^{*}$ & $-0.38^{\star}$ & $-0.21^{*}$ & $0 \cdot 40^{*}$ & $0.53^{*}$ & $0.50^{*}$ & $-0.32^{\star}$ & 1.00 \\
\hline
\end{tabular}

DQI-SNR, diet quality index based on the Swedish nutrition recommendations 2005.

${ }^{\star} P<0.01$.

tAdjusted for gender, age and season.

fDietary variables are expressed as percentage of energy (\%E) from non-alcohol energy intake (SFA; PUFA; sucrose), g/MJ (dietary fibre), g/week (fish and shellfish) or $\mathrm{g} / \mathrm{d}$ (fruit and vegetables).

Table 4 Population median intake and interquartile range of the specific DQI-SNR componentst, total energy intake and adjusted mean intakes§ of the DQI-SNR components by index score, among men ( $n$ 4525) and women ( $n$ 8491) of the Malmö Diet and Cancer cohort

\begin{tabular}{|c|c|c|c|c|c|c|c|c|c|c|}
\hline & \multirow[b]{2}{*}{ Median intake } & \multirow[b]{2}{*}{ IQR } & \multicolumn{7}{|c|}{ DQI-SNR score } & \multirow[b]{2}{*}{$P$ for trend } \\
\hline & & & 0 & 1 & 2 & 3 & 4 & 5 & 6 & \\
\hline \multicolumn{11}{|l|}{ MEN } \\
\hline Number of participants & & & 140 & 634 & 1462 & 1338 & 638 & 249 & 64 & \\
\hline Energy intake (MJ/d) & & & $10 \cdot 9$ & $10 \cdot 4$ & $10 \cdot 5$ & $10 \cdot 6$ & $10 \cdot 5$ & $10 \cdot 1$ & $10 \cdot 2$ & 0.081 \\
\hline SFA & $16 \cdot 3$ & $2 \cdot 1-38 \cdot 7$ & $19 \cdot 2$ & $18 \cdot 3$ & $17 \cdot 7$ & $16 \cdot 7$ & $14 \cdot 9$ & $13 \cdot 2$ & $12 \cdot 1$ & $<0.0001$ \\
\hline PUFA & $5 \cdot 9$ & $1 \cdot 8-15 \cdot 2$ & $4 \cdot 3$ & $5 \cdot 4$ & $6 \cdot 3$ & $6 \cdot 4$ & $6 \cdot 4$ & 6.5 & $6 \cdot 4$ & $<0.0001$ \\
\hline Fish and shellfish (g/week) & 291 & $0-3690$ & 125 & 190 & 267 & 425 & 485 & 503 & 673 & $<0.0001$ \\
\hline Dietary fibre (g/MJ) & $1 \cdot 9$ & $0 \cdot 5-8 \cdot 5$ & $1 \cdot 6$ & $1 \cdot 6$ & $1 \cdot 8$ & $2 \cdot 0$ & $2 \cdot 4$ & $2 \cdot 8$ & $2 \cdot 9$ & $<0.0001$ \\
\hline Fruit and vegetables $(\mathrm{g} / \mathrm{d})$ & 289 & $0-3859$ & 216 & 243 & 267 & 324 & 453 & 540 & 603 & $<0.0001$ \\
\hline Sucrose & $7 \cdot 8$ & $0 \cdot 5-35 \cdot 5$ & $13 \cdot 8$ & $10 \cdot 8$ & $8 \cdot 3$ & $7 \cdot 4$ & $7 \cdot 2$ & $7 \cdot 2$ & $6 \cdot 6$ & $<0.0001$ \\
\hline \multicolumn{11}{|l|}{ WOMEN } \\
\hline Number of participants & & & 315 & 1237 & 2482 & 2231 & 1377 & 668 & 156 & \\
\hline Energy intake (MJ/d) & & & $8 \cdot 4$ & $8 \cdot 4$ & $8 \cdot 1$ & $8 \cdot 3$ & $8 \cdot 1$ & $7 \cdot 9$ & $7 \cdot 9$ & $<0.0001$ \\
\hline SFA & $16 \cdot 2$ & $3 \cdot 6-36 \cdot 2$ & $18 \cdot 4$ & $18 \cdot 0$ & $17 \cdot 7$ & $16 \cdot 3$ & $15 \cdot 0$ & $13 \cdot 9$ & $12 \cdot 5$ & $<0.0001$ \\
\hline PUFA & $5 \cdot 6$ & $1 \cdot 9-15 \cdot 6$ & $4 \cdot 4$ & $5 \cdot 2$ & $5 \cdot 9$ & $6 \cdot 0$ & $6 \cdot 1$ & $6 \cdot 2$ & $6 \cdot 5$ & $<0.0001$ \\
\hline Fish and shellfish (g/week) & 254 & $0-2104$ & 143 & 170 & 231 & 330 & 359 & 446 & 525 & $<0.0001$ \\
\hline Dietary fibre (g/MJ) & $2 \cdot 2$ & $0 \cdot 5-7 \cdot 4$ & $1 \cdot 7$ & $1 \cdot 9$ & $2 \cdot 0$ & $2 \cdot 3$ & $2 \cdot 7$ & $2 \cdot 8$ & $2 \cdot 9$ & $<0.0001$ \\
\hline Fruit and vegetables $(\mathrm{g} / \mathrm{d})$ & 349 & $1-1871$ & 237 & 273 & 306 & 387 & 496 & 546 & 593 & $<0.0001$ \\
\hline Sucrose & $8 \cdot 5$ & $0 \cdot 7-36 \cdot 8$ & $13 \cdot 5$ & $10 \cdot 8$ & $8 \cdot 9$ & $8 \cdot 4$ & $8 \cdot 1$ & $7 \cdot 9$ & $7 \cdot 3$ & $<0.0001$ \\
\hline
\end{tabular}

DQI-SNR, diet quality index based on the Swedish nutrition recommendations 2005; IQR, interquartile range. tExpressed as non-alcohol energy percentage $(\% \mathrm{E})$ unless otherwise noted.

$\ddagger$ Adjusted for age and season.

§Adjusted for age, season and total energy intake. 
Table 5 Adjustedt mean macronutrient intakes (\%E) by categories of DQI-SNR score among men $(n$ 4525) and women ( $n$ 8491) of the Malmö Diet and Cancer cohort

\begin{tabular}{|c|c|c|c|c|}
\hline \multirow[b]{2}{*}{ Nutrient } & \multicolumn{3}{|c|}{ DQI-SNR score } & \multirow[b]{2}{*}{$P$ for trend } \\
\hline & $\begin{array}{c}\text { Low } \\
\text { (0 or } 1 \text { point) }\end{array}$ & $\begin{array}{c}\text { Medium } \\
\text { (2 or } 3 \text { points) }\end{array}$ & $\begin{array}{c}\text { High } \\
\text { (4-6 points) }\end{array}$ & \\
\hline \multicolumn{5}{|l|}{ MEN } \\
\hline Total fat & $39 \cdot 5$ & $40 \cdot 1$ & $35 \cdot 9$ & $<0 \cdot 0001$ \\
\hline MUFA & $13 \cdot 6$ & $14 \cdot 1$ & $12 \cdot 8$ & $<0.0001$ \\
\hline$n-6$ fatty acids & $4 \cdot 2$ & $5 \cdot 1$ & $5 \cdot 1$ & $<0.0001$ \\
\hline$n-3$ fatty acids & 0.8 & $1 \cdot 0$ & $1 \cdot 1$ & $<0.0001$ \\
\hline Total carbohydrates & $45 \cdot 9$ & $44 \cdot 2$ & $47 \cdot 3$ & $<0.0001$ \\
\hline Protein & $14 \cdot 5$ & $15 \cdot 7$ & $16 \cdot 8$ & $<0.0001$ \\
\hline Alcohol & $3 \cdot 6$ & $4 \cdot 6$ & $4 \cdot 1$ & $<0 \cdot 0001$ \\
\hline \multicolumn{5}{|l|}{ WOMEN } \\
\hline Total fat & $38 \cdot 6$ & $38 \cdot 8$ & $35 \cdot 3$ & $<0.0001$ \\
\hline MUFA & $13 \cdot 2$ & $13 \cdot 4$ & $12 \cdot 3$ & $<0.0001$ \\
\hline$n-6$ fatty acids & $4 \cdot 1$ & $4 \cdot 8$ & 4.9 & $<0.0001$ \\
\hline$n-3$ fatty acids & $0 \cdot 8$ & $1 \cdot 0$ & $1 \cdot 0$ & $<0.0001$ \\
\hline Total carbohydrates & $46 \cdot 3$ & $45 \cdot 0$ & $47 \cdot 6$ & $<0 \cdot 0001$ \\
\hline Protein & $15 \cdot 1$ & $16 \cdot 2$ & $17 \cdot 1$ & $<0.0001$ \\
\hline Alcohol & $2 \cdot 5$ & $3 \cdot 0$ & $2 \cdot 9$ & $<0 \cdot 0001$ \\
\hline
\end{tabular}

$\%$ E, percentage of energy; DQI-SNR, diet quality index based on the Swedish nutrition recommendations 2005. tAdjusted for age, total energy intake and season.

$\ddagger P$ for trend was determined using the DQI-SNR score in its continuous form and represents the $P$ value associated with the linear regression coefficient for the DQI-SNR score for continuous nutrient variables.

intakes across continuous total index score $<0 \cdot 0001$ ) and lower intakes of butter, high-fat dairy and meat, soft drinks, cakes and biscuits, and sweets and chocolate (all $P<0 \cdot 0001$ ). Men and women with a high DQI-SNR score were also more likely to reach the recommended intake levels for most micronutrients (Figs 2(a) and 2(b), respectively). However, although significantly higher intakes of vitamin $\mathrm{D}$ and folate $(P$ for trend $<0.0001$ ) were found for participants with a high score, most of the study population was below the recommended intake level for both vitamin D (82\%) and folate (98\%). In addition, most participants (close to $100 \%$ ) reached the recommended intake level for retinol equivalents, riboflavin, niacin equivalents, vitamin $\mathrm{B}_{6}$, vitamin $\mathrm{B}_{12}$ and $\mathrm{P}$.

Tables $6 a$ and $6 \mathrm{~b}$ show how selected characteristics of participating men and women are related to DQI-SNR score. Individuals with higher DQI-SNR score were more likely to be older, more physically active during leisure-time and at work, non-smokers, have a higher educational level and socio-economic status, and have changed their food habits in the past. Among women, energy misreporting was also associated with a higher score. In addition, a high score was associated with diabetes diagnosis and a previous cardiovascular event.

\section{Discussion}

The present study describes the development of a diet quality index based on the SNR 2005 and the SDG. Such an index could potentially assume several different designs. We designed the DQI-SNR to assess the key dietary factors that are thought to be related to development of chronic disease, under the prerequisite that these would jointly reflect overall diet quality.

One drawback of dietary indices is that it is possible only to compare individuals with high and low scores ${ }^{(10)}$. A large proportion of the study population was given an intermediate score (i.e. 2 or 3 points) and these individuals could have very different diets. However, intakes of the DQI-SNR components showed significant trends across total score demonstrating the index's capability of separating individuals based on intakes of the selected index components. Results also showed that index score was associated with intakes of several foods groups. Notably, a high score was associated with a diet in accordance with the SDG: choosing margarine instead of butter, high-fibre cereals, low-fat dairy and meat products, and consuming larger amounts of fruit and vegetables. Individuals with high scores also seemed to avoid or consume less high-fat dairy, soft drinks, cakes, biscuits, sweets and chocolate. In addition, index score was associated with intake of several macronutrients other than the components. Individuals with high scores seemed to meet the recommendations for vitamins and minerals to a greater extent, notably for vitamin D, vitamin $\mathrm{E}$, ascorbic acid, $\mathrm{K}, \mathrm{Mg}, \mathrm{Fe}, \mathrm{Zn}$ and Se. Only a small proportion of the total study population managed to reach recommended intake of vitamin D (18\%) and folate $(2 \%)$, which is in accordance with what is previously known about the nutritional status of Swedish populations ${ }^{(33,35,36)}$. We have also shown that the DQISNR score relates as expected to participant characteristics. For example, one would expect that individuals with higher age, non-smokers, high physical activity level and high educational and socio-economic status would 
(a)

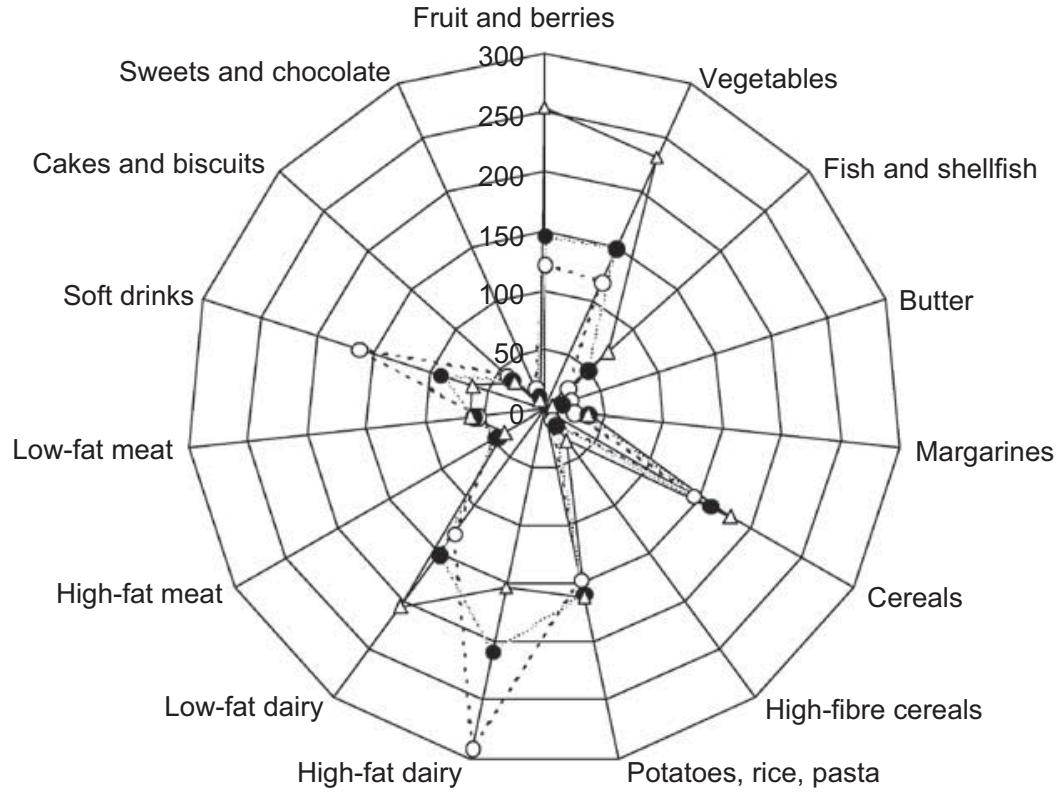

(b)

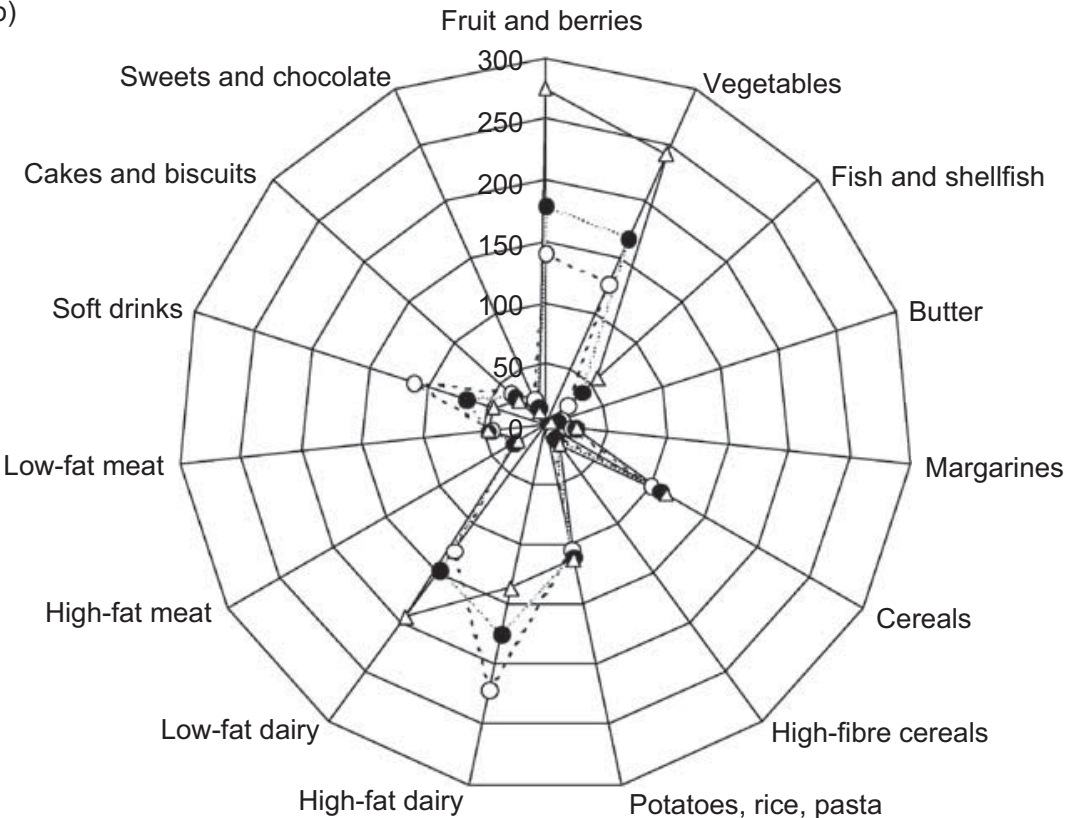

Fig. 1 Mean intake $(\mathrm{g} / \mathrm{d})$ of different food groups adjusted for energy intake, age and season in categories of DQI-SNR score (-- - --, low score (0 or 1 point); $\cdots \bullet$, medium score ( 2 or 3 points); $-\triangle-$, high score (4-6 points)) among (a) men ( $n 4525)$ and (b) women ( $n$ 8491) of the Malmö Diet and Cancer cohort. All $P$ for the linear trends of mean intake across total index score (continuous) were $<0.0001$ (DQI-SNR, diet quality index based on the Swedish nutrition recommendations 2005)

consume a higher-quality diet, as seen with the DQI-SNR. In addition, individuals reporting to have changed their food habits in the past (due to illness or other reason), having diabetes or having a previous cardiovascular event were more likely to have a higher total score. This is most likely a reflection of the fact that individuals who change their diets due to illness rely on the current dietary recommendations ${ }^{(55)}$. The associations seen with past food habit change and energy misreporting highlight the importance of excluding these individuals from analysis when investigating associations with disease endpoints.

Overall, the results of our study are consistent with the findings of previous studies using other indices in other populations ${ }^{(15,56-58)}$, as well as with other Swedish studies on dietary habits and nutritional status ${ }^{(33,36)}$.

Recently published reviews on diet quality indices highlighted several unresolved methodological issues of the index approach including the selection of components, 
(a)

Retinol equivalents

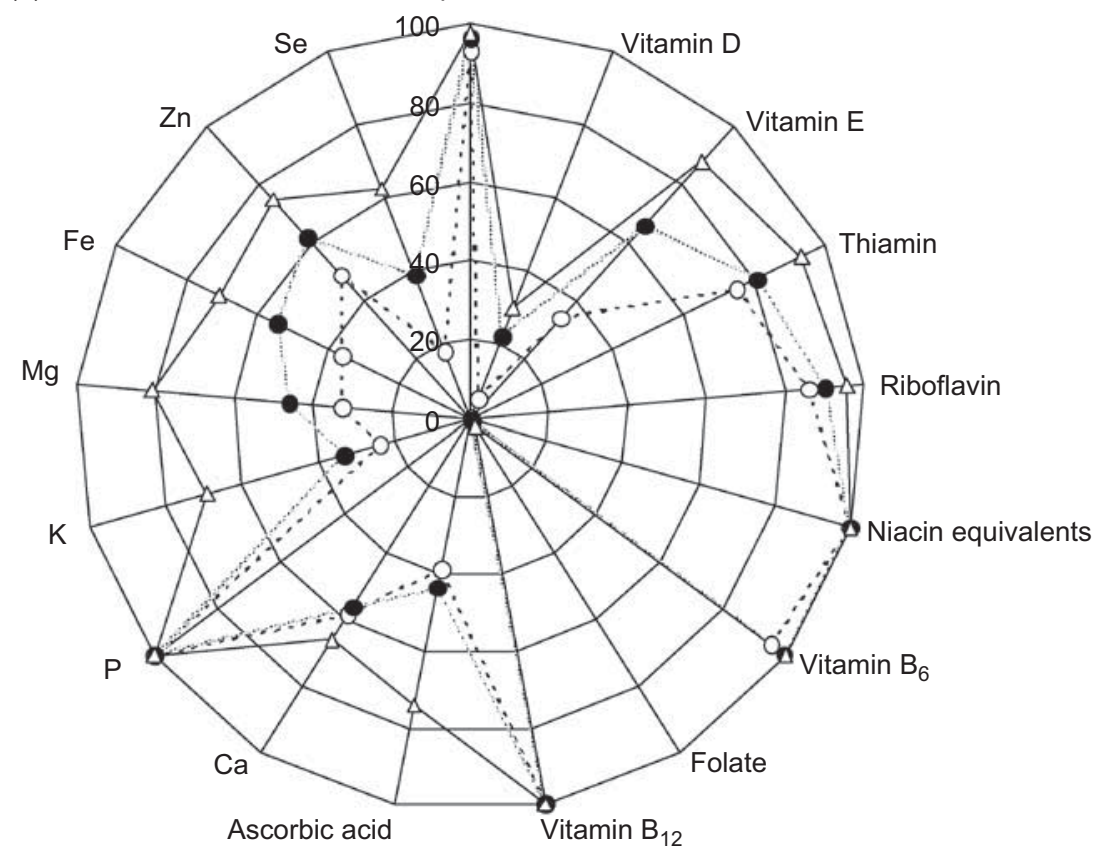

(b)

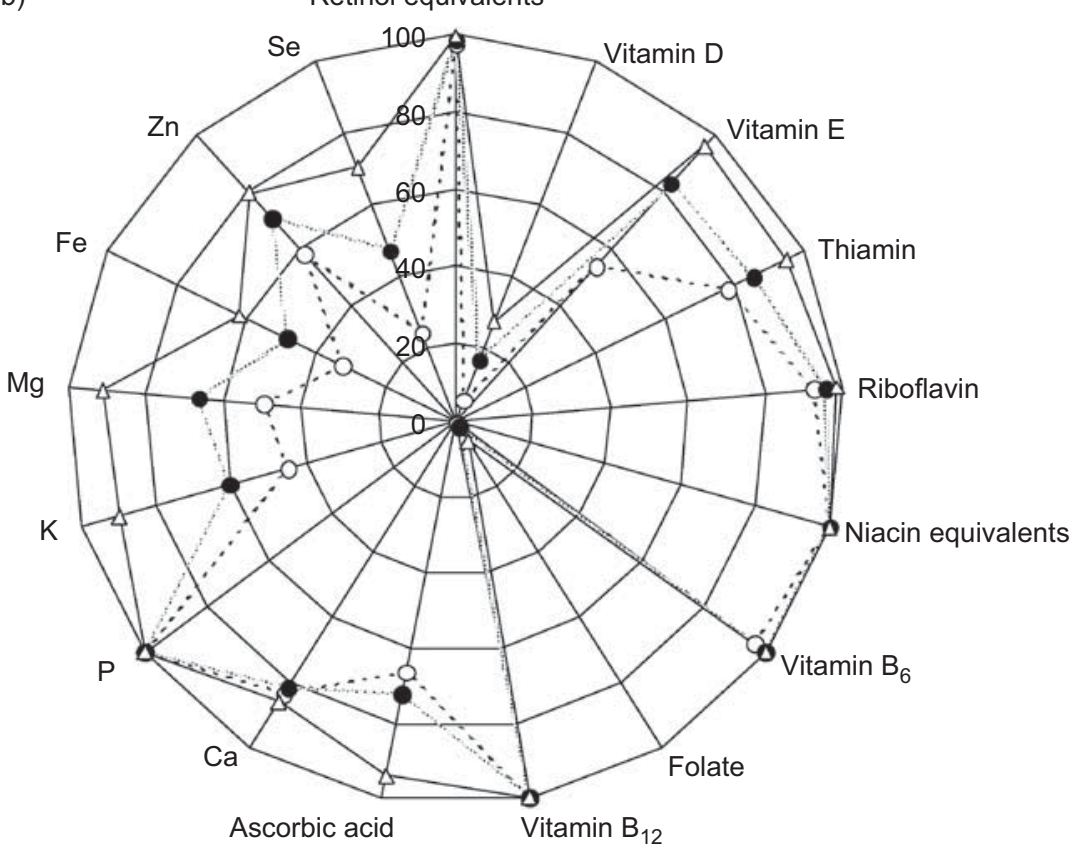

Fig. 2 Adherence (\%) to recommended intake level (g/MJ) of selected micronutrients in categories of DQI-SNR score (- - --- , low score (0 or 1 point); $\cdots \bullet$, medium score ( 2 or 3 points); $-\triangle-$, high score (4-6 points)) among (a) men ( $n$ 4525) and (b) women ( $n$ 8491) of the Malmö Diet and Cancer cohort (DQI-SNR, diet quality index based on the Swedish nutrition recommendations 2005)

inter-correlation between components, cut-off points and scoring. The subjective decisions made by the researcher regarding these issues may affect the diagnostic capacity of indices ${ }^{(10-12,14)}$

An important limitation of dietary indices is when some of the index items show high inter-correlation, which can result in some aspects of diet contributing greater weight to the overall index score ${ }^{(10,11)}$. What level of correlation is acceptable, however, is highly subjective and there are no methodological papers discussing this issue. The intercorrelation between components of the DQI-SNR was low, apart from a negative correlation between dietary fibre intake and SFA $(r=-0.54)$ and a positive correlation between dietary fibre and fruit and vegetables $(r=0.63)$. The correlation between dietary fibre and SFA is an example of an overlap in dietary behaviours that is inherent in the 
Table 6a Anthropometric, lifestyle and socio-economic factors, and disease history by categories of DQI-SNR score among men ( $n$ 4525) of the Malmö Diet and Cancer cohort

\begin{tabular}{|c|c|c|c|c|}
\hline & \multicolumn{3}{|c|}{ DQI-SNR score } & \multirow[b]{2}{*}{$P$ for trendt } \\
\hline & $\begin{array}{c}\text { Low } \\
\text { (0 or } 1 \text { point) }\end{array}$ & $\begin{array}{c}\text { Medium } \\
\text { (2 or } 3 \text { points) }\end{array}$ & $\begin{array}{c}\text { High } \\
\text { (4-6 points) }\end{array}$ & \\
\hline No. of participants & 774 & 2800 & 951 & \\
\hline Age (years) & $61 \cdot 5$ & $61 \cdot 8$ & $62 \cdot 3$ & $<0.0001$ \\
\hline BMI $\left(\mathrm{kg} / \mathrm{m}^{2}\right)$ & $25 \cdot 9$ & $26 \cdot 5$ & $26 \cdot 7$ & $<0.0001 \ddagger$ \\
\hline Waist circumference $(\mathrm{cm})$ & $92 \cdot 9$ & $94 \cdot 4$ & 93.9 & $0 \cdot 155 \ddagger$ \\
\hline Waist-to-hip ratio & 0.94 & 0.95 & 0.94 & $0.642 \ddagger$ \\
\hline High leisure-time physical activity (\%) & $29 \cdot 5$ & $32 \cdot 9$ & $37 \cdot 1$ & $<0.0001$ \\
\hline Sedentary work (\%) & $39 \cdot 3$ & $48 \cdot 0$ & $50 \cdot 2$ & $<0.0001$ \\
\hline Current smokers (\%) & $34 \cdot 4$ & $24 \cdot 5$ & $14 \cdot 2$ & $<0.0001$ \\
\hline Moderate/high alcohol consumption (\%) & $20 \cdot 7$ & $31 \cdot 2$ & $25 \cdot 2$ & 0.089 \\
\hline Past food habit change (\%) & $16 \cdot 3$ & $19 \cdot 8$ & $34 \cdot 7$ & $<0.0001$ \\
\hline Energy misreporters (\%) & $13 \cdot 4$ & $14 \cdot 6$ & $14 \cdot 8$ & 0.991 \\
\hline High educational level (\%) & $27 \cdot 5$ & $34 \cdot 0$ & $40 \cdot 6$ & $<0.0001$ \\
\hline High socio-economic status (\%) & $41 \cdot 3$ & $46 \cdot 7$ & $49 \cdot 7$ & $<0.0001$ \\
\hline Blood pressure $>140 / 90 \mathrm{mmHg}(\%)$ & $27 \cdot 4$ & $27 \cdot 4$ & $28 \cdot 2$ & $0.576 \S$ \\
\hline Previous cardiovascular event (\%) & $4 \cdot 8$ & $6 \cdot 7$ & $9 \cdot 4$ & $0.001 \S$ \\
\hline Diabetes diagnosis (\%) & $1 \cdot 8$ & $4 \cdot 3$ & $9 \cdot 3$ & $<0.0001 \S$ \\
\hline
\end{tabular}

DQI-SNR, diet quality index based on the Swedish nutrition recommendations 2005.

t $P$ for trend was determined using the DQI-SNR score in its continuous form and represents the $P$ value associated with the linear regression coefficient for the DQI-SNR score for continuous variables (age, BMI, waist, waist-to-hip ratio) and the logistic regression coefficient for the DQI-SNR score for dichotomous variables (high leisure-time physical activity, sedentary work, current smokers, moderate/high alcohol consumption, past food habit change, energy misreporting, high educational level, high socio-economic status, blood pressure, previous cardiovascular event and diabetes diagnosis). Analysis adjusted for age (except for mean age across score) and total energy intake, unless otherwise noted.

$\ddagger$ Adjusted for age, total energy intake and leisure-time physical activity.

§Adjusted for age, total energy intake, leisure-time physical activity and BMI.

Table 6b Anthropometric, lifestyle and socio-economic factors, and disease history by categories of DQI-SNR score among women ( $n$ 8491) of the Malmö Diet and Cancer cohort

\begin{tabular}{|c|c|c|c|c|}
\hline & \multicolumn{3}{|c|}{ DQI-SNR score } & \multirow[b]{2}{*}{$P$ for trendt } \\
\hline & $\begin{array}{c}\text { Low } \\
\text { (0 or } 1 \text { point) }\end{array}$ & $\begin{array}{c}\text { Medium } \\
\text { (2 or } 3 \text { points) }\end{array}$ & $\begin{array}{c}\text { High } \\
\text { (4-6 points) }\end{array}$ & \\
\hline No. of participants & 1552 & 4713 & 2201 & \\
\hline Age (years) & $57 \cdot 1$ & $57 \cdot 3$ & $58 \cdot 3$ & $<0.0001$ \\
\hline $\mathrm{BMI}\left(\mathrm{kg} / \mathrm{m}^{2}\right)$ & $25 \cdot 0$ & $25 \cdot 5$ & $25 \cdot 7$ & $<0.0001 \ddagger$ \\
\hline Waist circumference $(\mathrm{cm})$ & $77 \cdot 4$ & $78 \cdot 3$ & $78 \cdot 4$ & $0.004 \ddagger$ \\
\hline Waist-to-hip ratio & $0 \cdot 80$ & 0.81 & $0 \cdot 80$ & $0.579 \ddagger$ \\
\hline High leisure-time physical activity (\%) & $29 \cdot 3$ & $32 \cdot 2$ & $38 \cdot 3$ & $<0.0001$ \\
\hline Sedentary work (\%) & $41 \cdot 7$ & $43 \cdot 0$ & $42 \cdot 7$ & 0.484 \\
\hline Current smokers (\%) & $32 \cdot 7$ & $25 \cdot 5$ & $16 \cdot 0$ & $<0.0001$ \\
\hline Moderate/high alcohol consumption (\%) & $16 \cdot 0$ & $19 \cdot 5$ & $16 \cdot 4$ & $0 \cdot 123$ \\
\hline Past food habit change (\%) & $16 \cdot 5$ & $20 \cdot 7$ & $31 \cdot 9$ & $<0.0001$ \\
\hline Energy misreporters (\%) & $17 \cdot 8$ & $19 \cdot 1$ & $22 \cdot 7$ & 0.001 \\
\hline High educational level (\%) & $31 \cdot 7$ & $33 \cdot 4$ & $37 \cdot 9$ & $<0.0001$ \\
\hline High socio-economic status (\%) & $27 \cdot 9$ & $32 \cdot 7$ & 35.9 & $<0.0001$ \\
\hline Blood pressure $>140 / 90 \mathrm{mmHg}(\%)$ & $16 \cdot 0$ & $16 \cdot 8$ & $16 \cdot 2$ & $0.040 \S$ \\
\hline Previous cardiovascular event (\%) & $1 \cdot 4$ & 1.5 & $1 \cdot \overline{9}$ & $0.395 \$$ \\
\hline Diabetes diagnosis (\%) & $1 \cdot 2$ & $2 \cdot 0$ & $4 \cdot 5$ & $<0.0001 \S$ \\
\hline
\end{tabular}

DQI-SNR, diet quality index based on the Swedish nutrition recommendations 2005.

t $P$ for trend was determined using the DQI-SNR score in its continuous form and represents the $P$ value associated with the linear regression coefficient for the DQI-SNR score for continuous variables (age, BMI, waist, waist-to-hip ratio) and the logistic regression coefficient for the DQI-SNR score for dichotomous variables (high leisure-time physical activity, sedentary work, current smokers, moderate/high alcohol consumption, past food habit change, energy misreporting, high educational level, high socio-economic status, blood pressure, previous cardiovascular event and diabetes diagnosis). Analysis adjusted for age (except for mean age across score) and total energy intake, unless otherwise noted.

$\ddagger$ Adjusted for age, total energy intake and leisure-time physical activity.

$\S$ Adjusted for age, total energy intake, leisure-time physical activity and BMI.

SNR 2005 (i.e. individuals consuming low-fat and high-fibre diets). Since there is no specified recommendation for intake of wholegrain cereals, dietary fibre was included to reflect intake of wholegrain/fibre-rich cereals. However, the high correlation between fibre and fruit and vegetables suggests some degree of overlap which will have to be investigated in future studies using this index. Despite using energyadjusted components to a great extent, correlation with 
energy intake remained for some components as well as a very low correlation with total score. As other researchers have noted previously ${ }^{(59,60)}$, this suggests that the energy density method is not sufficient for complete energy adjustment. Therefore, it will be important to adjust for total energy intake in diet-disease analyses using the DQI-SNR.

Another potential limitation is the categorization of food and nutrient intake into dichotomies of 'compliance' and 'non-compliance'. Such categorization can conceal the true variability in the intake data and diminish the range of scores ${ }^{(10,14)}$. In the present study we opted for simplicity and used simple cut-offs for all components in order to evaluate adherence to the recommended intake levels. However, in order to provide sufficient discriminatory power for the SFA component, we had to add $1 \mathrm{SD}$ of the population mean to the cut-off value. In other study populations researchers should investigate the possibility of using the recommended intake as a cut-off (i.e. $<10 \% \mathrm{E}$ ). In addition, future studies need to examine the influence of each component as a continuous variable on disease outcome separately in the search for potential cut-offs with improved predictability.

Finally, the dietary assessment method used in the MDC is a diet history method, reflecting usual intake. Although the high relative validity of the method is a great advantage, it is not ideal for evaluating absolute intakes ${ }^{(39)}$. We therefore used relative intakes of most components, as well as for evaluation of nutrient intakes across total score. However, the fruit and vegetable component as well as the fish and shellifish component were based on absolute intakes ( $\mathrm{g} / \mathrm{d}$ or $\mathrm{g} /$ week) and thus the cut-offs for these components may not be appropriate. Previous studies within the MDC cohort have found that fruit and vegetable intake tends to be overreported $^{(41)}$.

Several dietary indices have previously been constructed. However, the Mediterranean Diet Score and the Healthy Diet Indicator for instance include food groups (e.g. olive oil and legumes) not consumed in large amounts in Swedish diets ${ }^{(22)}$. Also, no previous score has specifically addressed the Swedish dietary recommendations. In addition, few studies consider inter-correlation between components. For example, the Recommended Food Score developed by Kant et $a l .{ }^{(26)}$, which has previously been successfully applied in a Swedish cohort ${ }^{(61)}$, includes thirty-six recommended foods whereof a majority is fruit and vegetables, suggesting the possibility that this index is merely a reflection of fruit and vegetable intake rather than overall diet quality.

In conclusion, the results suggest that the DQI-SNR is a useful tool for assessing adherence to the SNR 2005 and the SDG. Future studies need to validate the DQI-SNR in relation to biochemical and clinical indicators of nutritional status, as well as assess its utility in assessing risk of mortality and morbidity. Also, the utility of the index needs to be examined in other population and age groups.

\section{Acknowledgements}

The study was supported by the Ernhold Lundström Foundation and Region Skåne. There are no conflicts of interest. I.D. is primary author of the paper and responsible for design, analysis and interpretation of data. All authors (I.D., B.G., U.E., E.S., J.N., P.W., B.H. and E.W.) contributed to the interpretation of data and finalisation of the manuscript. The authors are especially grateful to the men and women who participated in the Malmö Diet and Cancer Study.

\section{References}

1. Jacobs D \& Tapsell L (2007) Food, not nutrients, is the fundamental unit in nutrition. Nutr Rev 65, 439-450.

2. Tucker K (2001) Eat a variety of healthful foods: old advice with new support. Nutr Rev 59, 156-158.

3. Hoffman K, Schulze M \& Boeing H (2002) Dietary patterns: report of an international workshop. Public Health Nutr $\mathbf{5}$, 89-90.

4. Hu F (2002) Dietary pattern analysis: a new direction in nutritional epidemiology. Curr Opin Lipidol 13, 3-9.

5. Moeller S, Reedy J, Millen AE et al. (2007) Dietary patterns: challenges and opportunities in dietary pattern research an Experimental Biology workshop April 1, 2006. J Am Diet Assoc 107, 1233-1239.

6. Jacobs DR Jr, Gross M \& Tapsell L (2009) Food synergy: an operational concept for understanding nutrition. Am J Clin Nutr 89, 1543S-1548S.

7. Michels K \& Schulze M (2005) Can dietary patterns help us detect diet-disease associations? Nutr Res Rev 18, 241-248.

8. Newby P \& Tucker K (2004) Empirically derived eating patterns using factor or cluster analysis: a review. Nutr Rev 62, 177-203.

9. Kant A (2004) Dietary patterns and health outcomes. $J$ Am Diet Assoc 104, 615-635.

10. Waijers P, Feskens E \& Ocké M (2007) A critical review of predefined diet quality scores. BrJ Nutr 97, 219-231.

11. Arvaniti F \& Panagiotakos D (2008) Healthy indexes in public health practice and research: a review. Crit Rev Food Sci Nutr 48, 317-327.

12. Wirt A \& Collins C (2009) Diet quality - what is it and does it matter? Public Health Nutr 12, 2473-2492.

13. Kant A (1996) Indexes of overall diet quality: a review. J Am Diet Assoc 96, 785-791.

14. Kourlaba G \& Panagiotakos D (2009) Dietary quality indices and human health: a review. Maturitas 62, 1-8.

15. Kim S, Haines P, Siega-Riz A et al. (2003) The Diet Quality Index-International (DQI-I) provides an effective tool for cross-national comparison of diet quality as illustrated by China and the United States. J Nutr 133, 3476-3484.

16. Weinstein S, Vogt $\mathrm{T} \&$ Gerrior S (2004) Healthy Eating Index scores are associated with blood nutrient concentrations in the Third National Health and Nutrition Examination Survey. J Am Diet Assoc 104, 576-584.

17. Hann C, Rock C, King I et al. (2001) Validation of the Healthy Eating Index with use of plasma biomarkers in a clinical sample of women. Am J Clin Nutr 74, 479-486.

18. Neuhouser M, Patterson R, King I et al. (2003) Selected nutritional biomarkers predict diet quality. Public Health Nutr 6, 703-709.

19. Kant A \& Graubard B (2005) A comparison of three dietary pattern indexes for predicting biomarkers of diet and disease. J Am Coll Nutr 24, 294-303.

20. Fung T, Hu F, Barbieri R et al. (2007) Dietary patterns, the Alternate Healthy Eating Index and plasma sex hormone 
concentrations in postmenopausal women. Int $J$ Cancer 121, 803-809.

21. Boynton A, Neuhouser M, Wener M et al. (2007) Associations between healthy eating patterns and immune function or inflammation in overweight or obese postmenopausal women. Am J Clin Nutr 86, 1445-1455.

22. Huijbregts P, Feskens E, Räsänen L et al. (1997) Dietary pattern and 20 year mortality in elderly men in Finland, Italy, and the Netherlands: longitudinal cohort study. BMJ 315, 13-17.

23. Seymour J, Calle E, Flagg E et al. (2003) Diet Quality Index as a predictor of short-term mortality in the American Cancer Society Cancer Prevention Study II Nutrition Cohort. Am J Epidemiol 157, 980-988.

24. Osler M, Heitmann B, Gerdes L et al. (2001) Dietary patterns and mortality in Danish men and women: a prospective observational study. Br J Nutr 85, 219-225.

25. Knoops K, Groot de L, Fidanza F et al. (2006) Comparison of three different dietary scores in relation to 10 -year mortality in elderly European subjects: the HALE project. Eur J Clin Nutr 60, 746-755.

26. Kant A, Schatzkin A, Graubard B et al. (2000) A prospective study of diet quality and mortality in women. JAMA 283, 2109-2115.

27. McCullough M, Feskanich D, Stampfer M et al. (2002) Diet quality and major chronic disease risk in men and women: moving toward improved dietary guidance. Am J Clin Nutr 76, 1261-1271.

28. Alberti A, Fruttini D \& Fidanza F (2009) The Mediterranean Adequacy Index: further confirming results of validity. Nutr Metab Cardiovasc Dis 19, 61-66.

29. Fung T, Hu F, McCullough M et al. (2006) Diet quality is associated with the risk of estrogen receptor-negative breast cancer in postmenopausal women. J Nutr 136, 466-472.

30. Reedy J, Mitrou P, Krebs-Smith S et al. (2008) Index-based dietary patterns and risk of colorectal cancer. $A m J$ Epidemiol 168, 38-48.

31. Swedish National Food Administration (2005) Swedish Nutrition Recommendations 2005. Uppsala: National Food Administration.

32. Swedish National Food Administration (2010) Kostråd för vuxna (Dietary guidelines for adults). http://www.slv.se/sv/gruppa1/ mat-och-naring/kostrad/vuxna (accessed February 2010).

33. Nordic Council of Ministers (2004) Nordic Nutrition Recommendations 2004 - Integrating Nutrition and Physical Activity, 4th ed. Copenhagen: Nordic Council of Ministers.

34. Enghardt-Barbieri H \& Lindvall C (2003) Swedish Nutrition Recommendations Objectified (SNO) - Basis for General Advice on Food Consumption for Healthy Adults. Uppsala: Swedish National Food Administration.

35. Becker W \& Pearson M (2003) Riksmaten 1997-98. Dietary Habits and Nutrient Intake in Sweden 1997-98. The Second National Food Consumption Survey. Uppsala: Swedish National Food Administration.

36. Becker W (1998) Dietary guidelines and patterns of food and nutrient intake in Sweden. Br J Nutr 81, Suppl. 2, S113-S117.

37. >Berglund G, Elmståhl S, Janzon L et al. (1993) The Malmö Diet and Cancer study. Design and feasibility. J Intern Med 233, 45-51.

38. Manjer J, Carlsson S, Elmstahl S et al. (2001) The Malmö Diet and Cancer Study: representativity, cancer incidence and mortality in participants and non-participants. Eur J Cancer Prev 10, 489-499.

39. Wirfält E, Mattisson I, Johansson U et al. (2002) A methodological report from the Malmö Diet and Cancer Study: development and evaluation of altered routines in dietary data processing. Nutr J $\mathbf{1}, 3$.

40. Callmer E, Riboli E, Saracci R et al. (1993) Dietary assessment methods evaluated in the Malmö food study. J Intern Med 233, 53-57.
41. Elmståhl S, Riboli E, Lindegärde F et al. (1996) The Malmö food study: the relative validity of a modified diet history method and an extensive food frequency questionnaire for measuring food intake. Eur J Clin Nutr 50, 143-151.

42. Riboli E, Elmståhl S, Saracci R et al. (1997) The Malmö food study: validity of two dietary assessment methods for measuring nutrient intake. Int J Epidemiol 26, Suppl. 1, S161-S173.

43. Elmståhl S, Gullberg B, Riboli E et al. (1996) The Malmö food study: the reproducibility of a novel diet history method and an extensive food frequency questionnaire. Eur J Clin Nutr 50, 134-142.

44. Thiébaut ACM, Kipnis V, Schatzkin A et al. (2008) The role of dietary measurement error in investigating the hypothesized link between dietary fat intake and breast cancer - a story with twists and turns. Cancer Invest 26, 68-73.

45. Willett WC (1998) Nutrition Epidemiology, 2nd ed. New York: Oxford University Press.

46. Taylor H, Jacobs D, Schucker B et al. (1978) A questionnaire for the assessment of leisure time physical activities. J Chronic Dis 31, 741-755.

47. Richardson M, Leon A, Jacobs D et al. (1994) Comprehensive evaluation of the Minnesota Leisure Time Physical Activity Questionnaire. J Clin Epidemiol 47, 271-281.

48. Li C, Aronsson C, Hedblad B et al. (2009) Ability of physical activity measurements to assess health-related risks. Eur J Clin Nutr 63, 1448-1451.

49. Mattisson I, Wirfält E, Aronsson C et al. (2005) Misreporting of energy: prevalence, characteristics of misreporters and influence on observed risk estimates in the Malmö Diet and Cancer cohort. Br J Nutr 94, 832-842.

50. Goldberg G, Black A, Jebb S et al. (1991) Critical evaluation of energy intake using fundamental principles of energy physiology: 1. Derivation of cut-off limits to identify underreporting. Eur J Clin Nutr 45, 569-581.

51. Black A (2000) Critical evaluation of energy intake using the Goldberg cut-off for energy intake:basal metabolic rate. A practical guide to its calculation, use and limitations. Int J Obes Relat Metab Disord 24, 1119-1130.

52. Jakobsen M, Eilis J, Heitmann B et al. (2009) Major types of dietary fat and risk of coronary heart disease: a pooled analysis of 11 cohort studies. Am J Clin Nutr 89, 1425-1432.

53. Willett W, Howe G \& Kushi L (1997) Adjustment for total energy intake in epidemiologic studies. Am J Clin Nutr 65, Suppl. 4, 1220S-1228S.

54. Trochim W (2004) Research Methods Knowledge Base: Measurement Validity Types. http://www.socialresearchmethods.net/kb/measval.htm (accessed February 2010).

55. Sonestedt E, Wirfält E, Gullberg G et al. (2005) Past food habit change is related to obesity, lifestyle and socioeconomic factors in the Malmo Diet and Cancer Cohort. Public Health Nutr 8, 876-885.

56. Kennedy E, Ohls J, Carlson S et al. (1995) The Healthy Eating Index: design and applications. J Am Diet Assoc 95, 1103-1108.

57. Patterson R, Haines P \& Popkin B (1994) Diet quality index: capturing a multidimensional behavior. J Am Diet Assoc 94, 57-64.

58. Haines P, Siega-Riz A \& Popkin B (1999) The Diet Quality Index Revised: a measurement instrument for populations. J Am Diet Assoc 99, 697-704.

59. Kushi L, Sellers T, Potter J et al. (1992) Dietary fat and postmenopausal breast cancer. J Natl Cancer Inst 84, 1092-1099.

60. Mattisson I, Wirfält E, Gullberg B et al. (2001) Fat intake is more strongly associated with socio-economic characteristics, regardless of energy adjustment approach. Eur J Clin Nutr 55, 452-461.

61. Lauza J, Håkansson N, Brozozowska A et al. (2009) Diet quality and mortality: a population-based prospective study of men. Eur J Clin Nutr 63, 415-417. 